\title{
Stabilisation of in-plane periodic motion of electrodynamic tether system by combining tether length control and current control
}

\author{
Hirohisa Kojima* and Hidenori Aoki \\ Department of Aerospace Engineering, \\ Graduate School of System Design, \\ Tokyo Metropolitan University, \\ 6-6 Asahigaoka, Hino, Tokyo 191-0065, Japan \\ Email: hkojima@tmu.ac.jp \\ Email: aokoyaka@gmail.com \\ *Corresponding author
}

\begin{abstract}
A tether system is a large flexible structure connecting two or more satellites. Libration control methods of a tether system in elliptic orbits have been studied. For electrodynamic tether systems, these have included control of the tether length and the electric current. In previous studies, these two control methods were considered independently. However, by combining them, it is expected that the periodic motion of an electrodynamic tether system can be stabilised using a smaller electric current. The tether satellite system considered in the present study consists of two subsatellites and a mother satellite connected together in series via massless tethers. To stabilise the periodic motion using only a small electric current, two different tethers are used. The first is a non-electrodynamic tether whose length acceleration is varied using bang-bang control, and the other is an electrodynamic tether subject to proportional-derivative or delayed feedback control. The results of numerical simulations show that the proposed control scheme exhibits good performance for stabilising librational motion of the tether system to periodic motion, combining non-electrodynamic and electrodynamic tethers.
\end{abstract}

Keywords: electrodynamic tether; EDT; periodic motion; PD control; delayed feedback control.

Reference to this paper should be made as follows: Kojima, H. and Aoki, H. (2015) 'Stabilisation of in-plane periodic motion of electrodynamic tether system by combining tether length control and current control', Int. J. Space Science and Engineering, Vol. 3, No. 4, pp.318-341.

Biographical notes: Hirohisa Kojima is a Professor at the Department of Aerospace Engineering, Tokyo Metropolitan University. He received his BE, $\mathrm{ME}$ and $\mathrm{PhD}$ from the Department of Aeronautics and Astronautics, the University of Tokyo. His current research interests are dynamics and control of space robots, optimisation, and chaos control. He is a member of JSASS, $J S M E$, SICE, IEEE, a senior member of AIAA, and a member of RSJ. He is on the Editorial Board of the JSASS and the International Journal of Space Science and Engineering. 
Hidenori Aoki received his Bachelor and Master degrees in Engineering from the Department of Aerospace Engineering, Tokyo Metropolitan University, Japan in 2013 and 2015, respectively.

This paper is a revised and expanded version of a paper entitled 'Periodic motion stabilization of electrodynamic tether system by combining tether length control and current control' presented at 29th International Symposium on Space Technology and Science, Nagoya, June 2-9, 2013.

\section{Introduction}

A tether system is a large flexible structure connecting a plurality of satellites by tether-like cord elements in space. Tether systems, from the viewpoints of weight reduction and deployability, are expected to be an important infrastructure for future space missions such as high-altitude atmospheric observations, orbital transfer and debris removal (Peláez and Lara, 2003; Peláez and Lorenzini, 2005; Peláez and Andres, 2005; Williams, 2006; Kojima et al., 2004, 2011a, 2011b; Kojima and Sugimoto, 2009, 2010; Nakanishi et al., 2011). In particular, electrodynamic tether (EDT) systems are expected to act as more efficient thrusters than traditional chemical thrusters because they consume less propellant by using the Lorentz force caused by interaction between the Earth's magnetic field and an electric current in a conductive tether. In tether satellite systems in elliptic orbits, no equilibrium state exists due to changes in the orbital angular velocity and the direction of gravity with respect to the tether satellite orientation. It is known from the results of numerical simulations and analysis of the Lyapunov exponent that tethered satellite systems irregularly swing with respect to the mother satellite in elliptic orbits with eccentricities of less than 0.3. The amplitude of this chaotic libration motion increases as the eccentricity increases, and the subsatellite starts rotating (tumbling) around the mother satellite when the eccentricity reaches around 0.32 (Karasopoulos and Richardson, 1992; Fujii et al., 2000). Various methods have been proposed for suppressing such chaotic librational motion, such as control of the tether tension (Kojima et al., 2011a), the tether attachment point, and the Lorentz force produced by a current in an EDT system (Peláez and Lara, 2003; Peláez and Lorenzini, 2005; Peláez and Andres, 2005; Williams, 2006; Kojima et al., 2004; Kojima and Sugimoto, 2009, 2010; Iñarrea and Peláez, 2010; Iñarrea et al., 2014). In addition, because delayed feedback control (Pyragas, 1992) (a method of chaos control in which a periodic solution is not referred to for the control input) is highly robust against parameter errors, it is suitable for motion control of a chaotic EDT system (Peláez and Lorenzini, 2005; Kojima et al., 2004; Kojima and Sugimoto, 2009, 2010; Iñarrea and Peláez, 2010; Iñarrea et al., 2014).

In previous studies, control of the tether length and electric current in an EDT system were considered independently. However, by combining these two control methods, it is expected that periodic motion can be stabilised either more rapidly or using a smaller electric current. This is the main objective of the present study. The tether satellite system considered consists of two subsatellites and a mother satellite connected together in series via massless tethers. The system uses both a non-EDT, whose length acceleration is subject to bang-bang control, and an EDT, which is subject to either 
proportional-derivative (PD) or delayed feedback (DF) control. Since the geomagnetic Lorentz force increases with decreasing distance to the centre of the Earth, the length of the non-EDT is controlled such that it is deployed from perigee to apogee and retrieved from apogee to perigee; in this way, an effective Lorentz force is obtained even at apogee.

\section{Model description}

\subsection{System model}

To simplify the analysis, gravity and the Lorentz force are considered to be the only external forces that affect the tethered satellite system. The centre of mass of the system follows an elliptical orbit on the equatorial plane. A schematic model of the EDT system treated is illustrated in Figure 1. This system consists of a mother satellite, two subsatellites, and two tethers. The mother satellite and the two subsatellites are labelled 0,1 , and 2 , and are treated as point masses of $m_{0}, m_{1}$, and $m_{2}$, respectively. The mother satellite is connected to subsatellite 1 by tether 1 , and subsatellite 1 is connected to subsatellite 2 by tether 2 . Although in reality, a tether will have non-zero mass and flexibility, for simplicity in the present study, tethers 1 and 2 are assumed to be rigid bodies without mass or inertia. In other words, subsatellite 1, which is located between the mother satellite and subsatellite 2, can be interpreted as the tether mass. Tether 1 is non-conductive but deployable, whereas tether 2 is conductive, and its length is constant. The direction of positive electric current is defined as the direction from subsatellite 1 to subsatellite 2. The origin of the orbital reference frame is the centre of the mother satellite. In this reference frame, the $x$-axis runs from the centre of the Earth to the centre of mass of the mother satellite, the $z$-axis runs along the angular momentum vector of the system, and the $y$-axis is the cross product of the $z$ - and $x$-axes. Vectors $\boldsymbol{i}, \boldsymbol{j}$, and $\boldsymbol{k}$ are unit vectors along the $x$-, $y$-, and $z$-axes, respectively. Because the mass of the mother satellite is assumed to be much greater than that of the subsatellites, the centre of mass of the system is coincident with the mother satellite. Furthermore, we consider only in-plane motion of the EDT system.

Figure 1 Three-mass tether system model

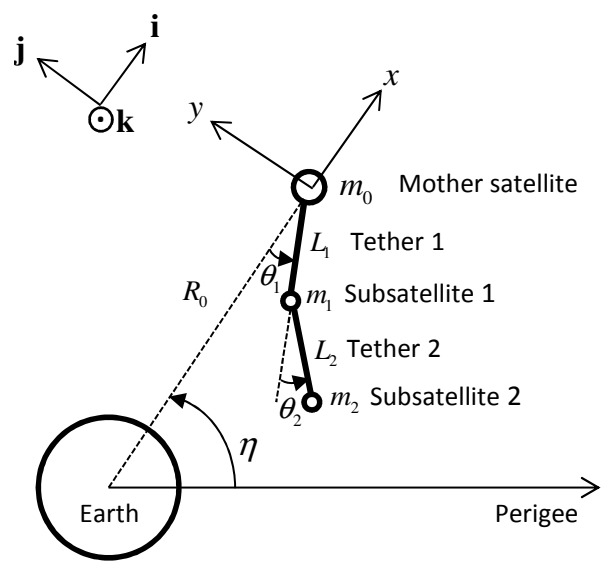




\subsection{Electrodynamic force}

In order to simplify the analyses, a non-tilted dipole model is used to describe the Earth's magnetic field, and the origin of the geocentric inertial frame is assumed to be the centre of mass of the Earth (Hughes, 1986). The magnetic field at the centre of tether 2 in the geocentric inertial frame is given by

$$
\boldsymbol{B}=-\frac{\mu_{m}}{R_{m}^{3}}\left[\begin{array}{c}
3 \sin \phi_{m} \cos \phi_{m} \cos \theta_{m} \\
3 \sin \phi_{m} \cos \phi_{m} \sin \theta_{m} \\
3 \sin ^{2} \phi_{m}-1
\end{array}\right]
$$

where $R_{m}$ is the orbital radius of the centre of tether 2. Since a non-tilted dipole model is assumed for the Earth's magnetic field and only in-plane motion of the EDT system is considered, the magnetic field can be reduced to

$$
\boldsymbol{B}=\left[\begin{array}{lll}
0 & 0 & \frac{\mu_{m}}{R_{m}^{3}}
\end{array}\right]^{T} .
$$

The electrodynamic force exerted on tether 2 is given in the geocentric inertial reference frame by

$$
\boldsymbol{F}^{e}=L_{2}(\boldsymbol{I} \times \boldsymbol{B}) .
$$

For simplicity, we divide the above electrodynamic force into the components that affect subsatellites 1 and 2 as follows:

$$
\boldsymbol{F}_{1}^{e}=\boldsymbol{F}_{2}^{e}=\boldsymbol{F}^{e} / 2 .
$$

\subsection{Equations of motion}

The parameters $R_{0}, L_{1}, \eta, \theta_{1}$, and $\theta_{2}$ are considered to be generalised coordinates, and their time-derivatives are then taken to be generalised speeds. The velocity $\boldsymbol{v}_{i}$ and acceleration $\boldsymbol{a}_{i}$ of the $i^{\text {th }}$ satellite in the geocentric inertial reference frame are obtained, respectively, as

$$
\begin{aligned}
\boldsymbol{v}_{i} & =\dot{\boldsymbol{R}}_{i}+\boldsymbol{\omega} \times \boldsymbol{R}_{i}, \\
\boldsymbol{a}_{i} & =\ddot{\boldsymbol{R}}_{i}+\dot{\boldsymbol{\omega}} \times \boldsymbol{R}_{i}+2 \boldsymbol{\omega} \times \dot{\boldsymbol{R}}_{i}+\boldsymbol{\omega} \times\left(\boldsymbol{\omega} \times \boldsymbol{R}_{i}\right)
\end{aligned}
$$

where $\boldsymbol{\omega}=\dot{\eta} \boldsymbol{k}$. The gravitational force on each satellite is

$$
\boldsymbol{F}_{i}^{g}=-\mu_{e} m_{i} \frac{\boldsymbol{R}_{i}}{\left|R_{i}\right|^{3}}, \quad(i=0,1,2) .
$$

The active force vector related to the tether tension $T$ is

$$
\boldsymbol{F}^{t}=T\left[\begin{array}{c}
\cos \theta_{1} \\
\sin \theta_{1} \\
0
\end{array}\right]
$$


The partial velocity vector for the generalised speed is

$$
\boldsymbol{v}_{i j}=\frac{\partial \boldsymbol{v}_{i}}{\partial q_{j+5}}, \quad(j=1,, \ldots, 5) .
$$

Using Kane's equation,

$$
\begin{aligned}
K_{j}^{*} & =-m_{0} \ddot{\boldsymbol{R}}_{0} \cdot \boldsymbol{v}_{0 j}-m_{1} \ddot{\boldsymbol{R}}_{1} \cdot \boldsymbol{v}_{1 j}-m_{2} \ddot{\boldsymbol{R}}_{2} \cdot \boldsymbol{v}_{2 j}, \\
K_{j} & =\boldsymbol{F}_{0}^{g} \cdot \boldsymbol{v}_{0 j}+\left(\boldsymbol{F}_{1}^{g}+\boldsymbol{F}_{1}^{e}+\boldsymbol{F}^{t}\right) \cdot \boldsymbol{v}_{1 j}+\left(\boldsymbol{F}_{2}^{g}+\boldsymbol{F}_{2}^{e}\right) \cdot \boldsymbol{v}_{2 j},
\end{aligned}
$$

the equations of motion can be obtained as

$$
\boldsymbol{M}(\boldsymbol{q}) \frac{d^{2}}{d t^{2}}\left[\begin{array}{c}
R_{0} \\
L_{1} \\
\eta \\
\theta_{1} \\
\theta_{2}
\end{array}\right]=\boldsymbol{f}(\boldsymbol{q})+\boldsymbol{g}(\boldsymbol{q}) \boldsymbol{u}
$$

where $M$ is the mass matrix $\in \mathcal{R}^{5 \times 5}, f$ is the nonlinear term vector $\in \mathcal{R}^{5}, g$ is the linear matrix $\in \mathcal{R}^{5 \times 2}$, and $\boldsymbol{u}$ is the control input vector defined as

$$
\boldsymbol{u}=\left[\begin{array}{l}
u_{1} \\
u_{2}
\end{array}\right]=\left[\begin{array}{c}
T \\
F^{e}
\end{array}\right]
$$

Although the electrodynamic force depends on the magnitude of the magnetic field and the electric current, for simplicity, we assume that the magnitude of the electrodynamic force is directly determined by PD or DF control. Instead, the electric current is calculated from the electrodynamic force and the magnetic field, which depends on the orbital radius of the centre of tether 2 .

Since we focus on the librational motion of the EDT system, the output variable vector is taken to be

$$
\boldsymbol{y}=\left[\begin{array}{l}
\theta_{1} \\
\theta_{2}
\end{array}\right] .
$$

The Mathematica code used to derive equation (12) is given in Appendix A, and the elements of the mass matrix $M$ and the nonlinear terms $f$ and $g$ are given in Appendix B.

The acceleration of the state variables is usually determined by substituting the state variable values and the control input into the equations of motion and solving the equations with respect to the acceleration of the state variables. On the other hand, because we assume in this study that the acceleration of $L_{1}$ is under bang-bang control, the acceleration and speed of $L_{1}$ are prescribed, and are not determined from the equations of motion. Instead, the tether tension is treated as an unknown variable. The tether tension and the acceleration of the state variables (except $L_{1}$ ) are then determined by substituting the state variable values, the acceleration and speed of $L_{1}$, and the 
electrodynamic force into the equations of motion. This inverse calculation of the tether tension can be explained as follows. First, equation (12) is rewritten as:

$$
\begin{aligned}
& {\left[\boldsymbol{M}_{1}(\boldsymbol{q}), \boldsymbol{M}_{2}(\boldsymbol{q}), \boldsymbol{M}_{3}(\boldsymbol{q}), \boldsymbol{M}_{4}(\boldsymbol{q}), \boldsymbol{M}_{5}(\boldsymbol{q})\right]\left[\begin{array}{c}
\ddot{R} \\
\ddot{L}_{1} \\
\ddot{\eta} \\
\ddot{\theta}_{1} \\
\ddot{\theta}_{2}
\end{array}\right]} \\
& =\boldsymbol{f}(\boldsymbol{q})+\left[\boldsymbol{g}_{1}(\boldsymbol{q}), \boldsymbol{g}_{2}(\boldsymbol{q})\right]\left[\begin{array}{c}
T \\
F^{e}
\end{array}\right]
\end{aligned}
$$

where $\boldsymbol{M}_{i},(i=1 \cdots 5)$ are column vectors of $\boldsymbol{M}$, and $\boldsymbol{g}_{i},(i=1,2)$ are column vectors of $\boldsymbol{g}$. Because the acceleration of $L_{1}\left(\ddot{L}_{1}\right)$ is prescribed and the tether tension $T$ is treated as an unknown variable that is determined from the equation of motion, we convert equation (15) to

$$
\boldsymbol{M}^{\prime}(\boldsymbol{q})\left[\begin{array}{c}
\ddot{R} \\
T \\
\ddot{\eta} \\
\ddot{\theta}_{1} \\
\ddot{\theta}_{2}
\end{array}\right]=\boldsymbol{f}(\boldsymbol{q})+\boldsymbol{g}_{2}(\boldsymbol{q}) F^{e}-\boldsymbol{M}_{2}(\boldsymbol{q}) \ddot{L}_{1} .
$$

where $\boldsymbol{M}^{\prime}(\boldsymbol{q})=\left[\boldsymbol{M}_{1}(\boldsymbol{q}),-\boldsymbol{g}_{1}(\boldsymbol{q}), \boldsymbol{M}_{3}(\boldsymbol{q}), \boldsymbol{M}_{4}(\boldsymbol{q}), \boldsymbol{M}_{5}(\boldsymbol{q})\right]$. Using the above equation, the acceleration of the state variables (except $L_{1}$ ) and the tether tension can be calculated as follows:

$$
\left[\begin{array}{c}
\ddot{R} \\
T \\
\ddot{\eta} \\
\ddot{\theta}_{1} \\
\ddot{\theta}_{2}
\end{array}\right]=\boldsymbol{M}^{\prime}(\boldsymbol{q})^{-1}\left(\boldsymbol{f}(\boldsymbol{q})+\boldsymbol{g}_{2}(\boldsymbol{q}) F^{e}-\boldsymbol{M}_{2}(\boldsymbol{q}) \ddot{L}_{1}\right) \text {. }
$$

\section{PD control of electrodynamic force}

\subsection{PD control}

In order to obtain the Lorentz force acting on the tether system, we consider a PD-controlled electrodynamic force input as follows:

$$
F^{e}=K_{P} \theta_{1}+K_{D} \dot{\theta}_{1} .
$$

PD control is typically used to damp out oscillations or vibrations based on the magnitude and speed of the controlled variable. In this study, however, it is used to stabilise the periodic librational motion of a tether system in an elliptic orbit. It should also be noted that the above electrodynamic force is determined by the PD controller based on the angle and angular speed of tether 1, but is applied to conductive tether 2, and the sign of the controller is opposite to that of a typical PD controller. This is because in the present study, a positive electrodynamic force acts in a direction that damps out tether libration. 


\subsection{Numerical results for PD control}

The parameters used in the numerical simulations were as follows. The initial conditions for the tether system were $R_{0}=6,600 \mathrm{~km}, \eta=0, \theta_{1}=0 \mathrm{rad}, \theta_{2}=0 \mathrm{rad}, \dot{R}_{0}=0 \mathrm{~km} / \mathrm{s}$, $\dot{\eta}=1.2899 \times 10^{-3} \mathrm{rad} / \mathrm{s}$, and $\dot{\theta}_{1}=\dot{\theta}_{2}=0 \mathrm{rad} / \mathrm{s}$. The orbital period was $7,457 \mathrm{~s}$, and the eccentricity of the centre of mass of the system was 0.2 .

When choosing control gains, factors such as ensuring a positive tether tension and a reasonable electric current should be taken into consideration. However, for simplicity, these factors were ignored and PD control gains of $K_{P}=0.01$ and $K_{D}=10.0$ were chosen based on trial calculations. Although these values were not always optimal, as shown later, they were sufficient to stabilise librational motion of the tether system and produce periodic motion.

We studied three cases with respect to $L_{1}: 50 \mathrm{~km}$ (Case-A), varying between 50 and $60 \mathrm{~km}$ (Case-B), and varying between 50 and $70 \mathrm{~km}$ (Case-C). In many previous studies on tether deployment control, a tether length of $100 \mathrm{~km}$ was assumed. Therefore, in the present study, we assumed a nominal total tether length of $L_{1}+L_{2}=100 \mathrm{~km}$. In order to investigate the effect of the tether length on the performance of periodic librational motion control, we allowed $L_{1}$ to vary by $10 \%$ or $20 \%$ of the total tether length, corresponding to 10 or $20 \mathrm{~km}$, respectively. In the case of a variation of $20 \mathrm{~km}$, even if the mother satellite fails to retrieve this length of tether, and $L_{1}$ remains at $70 \mathrm{~km}$, the altitude of subsatellite 2 at perigee will not be below $100 \mathrm{~km}$, where significant air drag occurs, because the total tether length will be $120 \mathrm{~km}$ whereas the altitude of the mother satellite at perigee is $222 \mathrm{~km}$.

The time responses of the tether length, tether angles, tether tension, and electric current in tether 2 for Case-A are shown in Figures 2 to 5, respectively. Figures 6 to 9 show the corresponding results for Case-B. As shown in Figure 6, tether 1 is deployed from perigee to apogee and retrieved from apogee to perigee, as described in Section 1. Figures 10 to 13 show the time responses of the tether length, tether angles, tether tension, and electric current in tether 2, respectively, for Case-C. It can be seen that the range of $L_{1}$ is larger, $\theta_{1}$ is smaller, and the amplitude of $\theta_{2}$ is slightly larger than for Case-B. In addition, when the range of $L_{1}$ increases, the electric current decreases, and the minimum tether tension increases. This is because the Earth's magnetic field and gravity gradient are larger when the subsatellite is at a lower altitude, so that the electric current required to stabilise the periodic motion becomes smaller, and the tether tension becomes larger.

The results of the numerical simulations show that the librational motion is stabilised to periodic motion after one orbit for all three cases of PD control. This convergence time is much shorter than that for DF control, as will be shown later. On the other hand, the electric current for PD control is much larger than that for DF control, as will also be described later. Although it is of interest to investigate the trade-off relationship among the magnitude of the electric current, the control gains and the convergence time, this is outside the scope of the present study, and will be a subject for future work.

The time response of the electric current is most closely correlated with $\theta_{1}$, and shows only a slight dependence on $\theta_{2}$. This is because the electric current is reversely determined by the magnitude of the Lorentz force and the magnetic field vector at the centre of tether 2 , which mainly depend on $\theta_{1}$. 
Figure 2 Time response of tether length for $L_{1}=50 \mathrm{~km}$

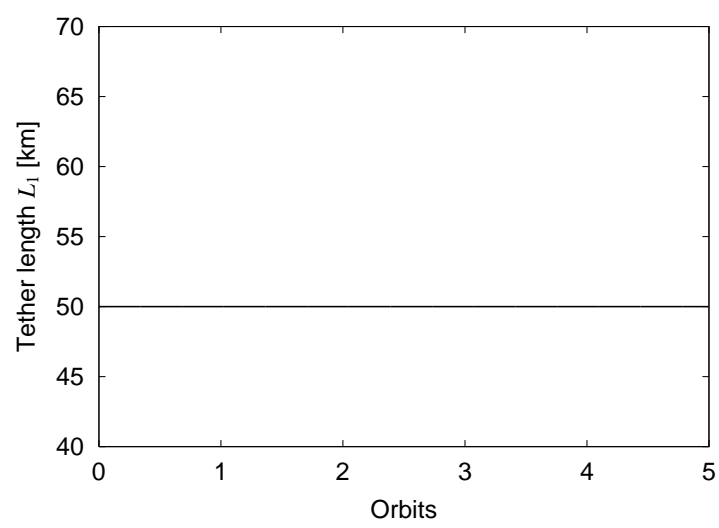

Figure 3 Time response of tether angles for PD control and $L_{1}=50 \mathrm{~km}$

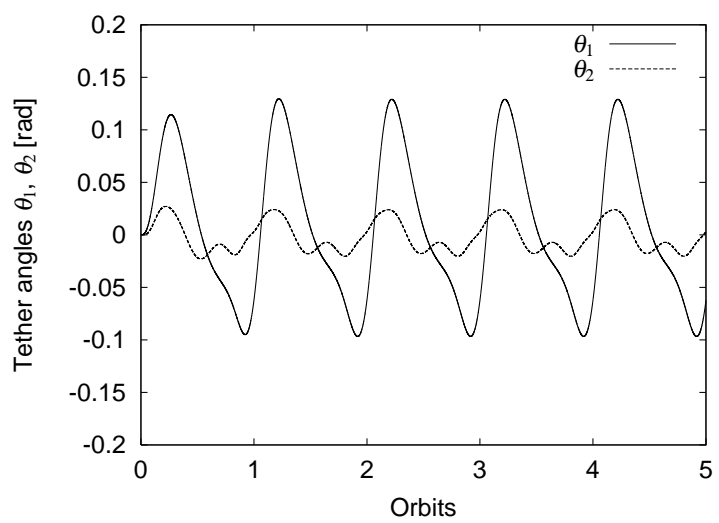

Figure 4 Time response of tether tension for PD control and $L_{1}=50 \mathrm{~km}$

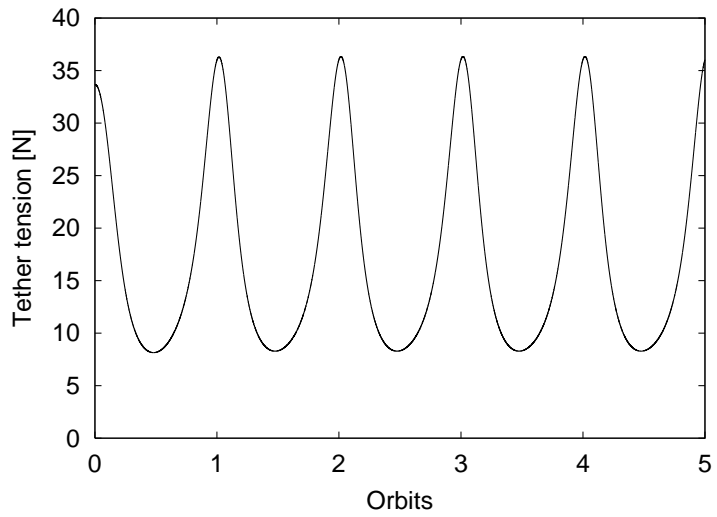


Figure 5 Time response of electric current for PD control and $L_{1}=50 \mathrm{~km}$

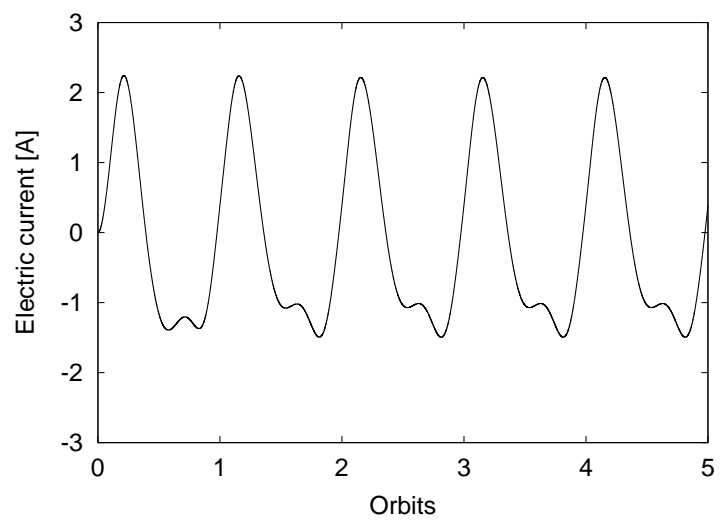

Figure 6 Time response of tether length for $L_{1}=50-60 \mathrm{~km}$

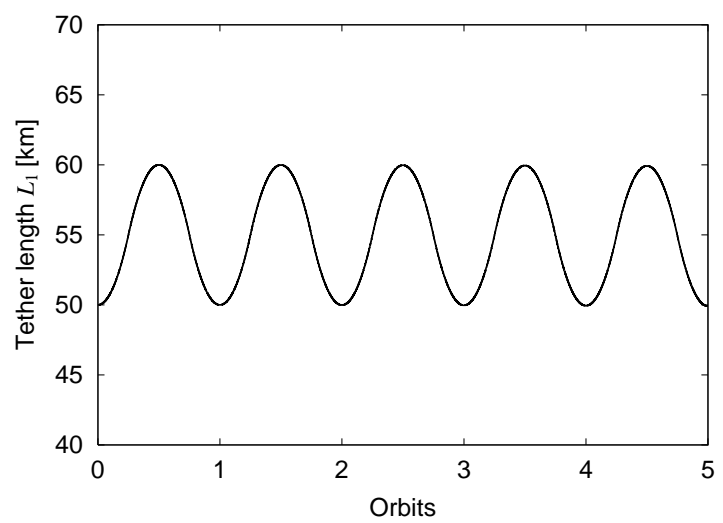

Figure 7 Time response of tether angles for PD control and $L_{1}=50-60 \mathrm{~km}$

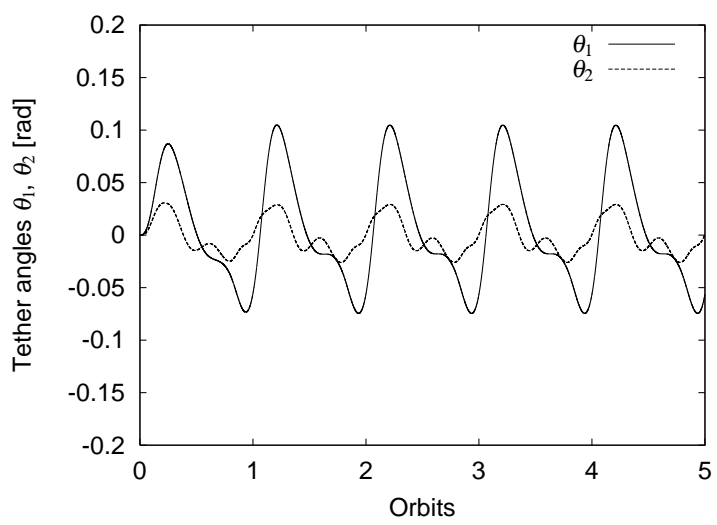


Figure 8 Time response of tether tension for PD control and $L_{1}=50-60 \mathrm{~km}$

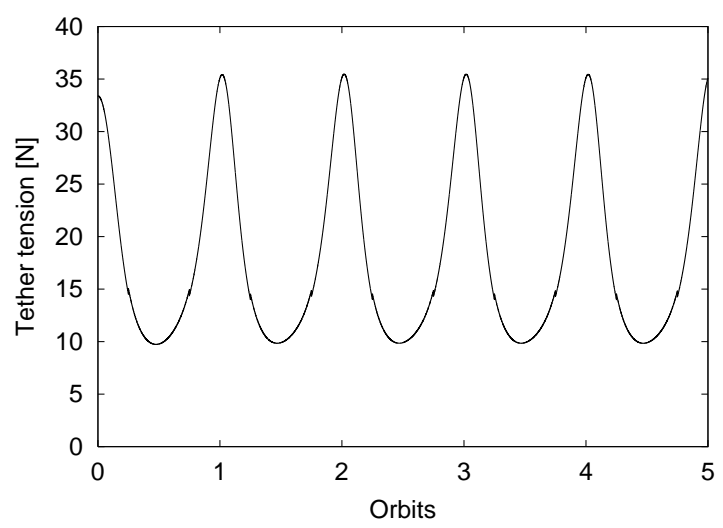

Figure 9 Time response of electric current for PD control and $L_{1}=50-60 \mathrm{~km}$

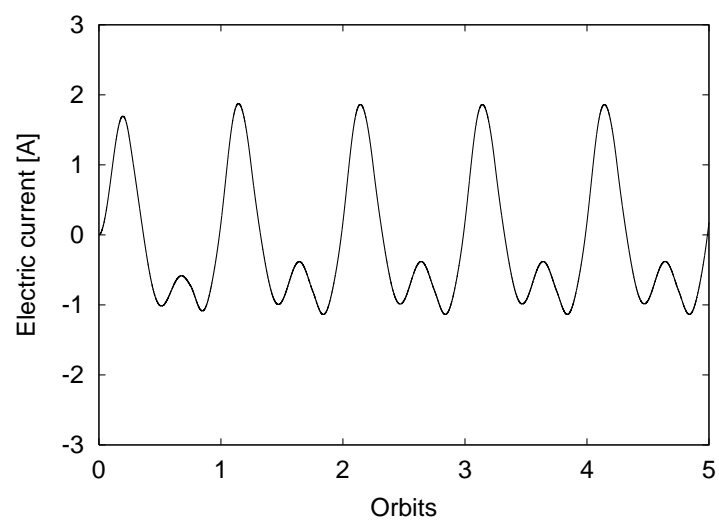

Figure 10 Time response of tether length for $L_{1}=50-70 \mathrm{~km}$

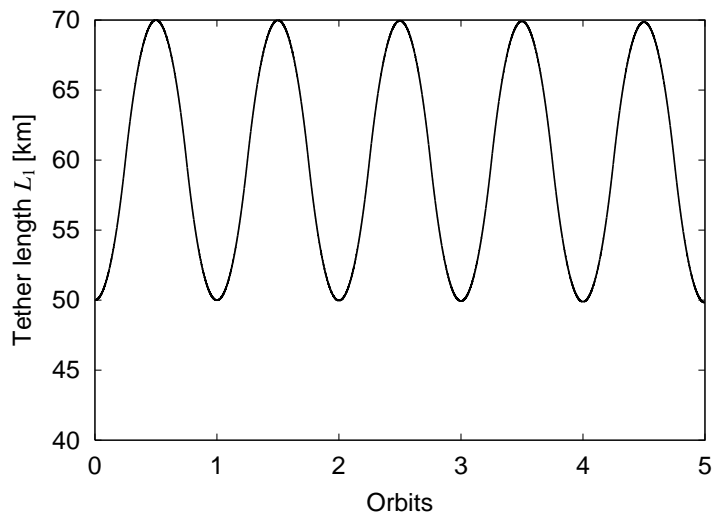


Figure 11 Time response of tether angles for PD control and $L_{1}=50-70 \mathrm{~km}$

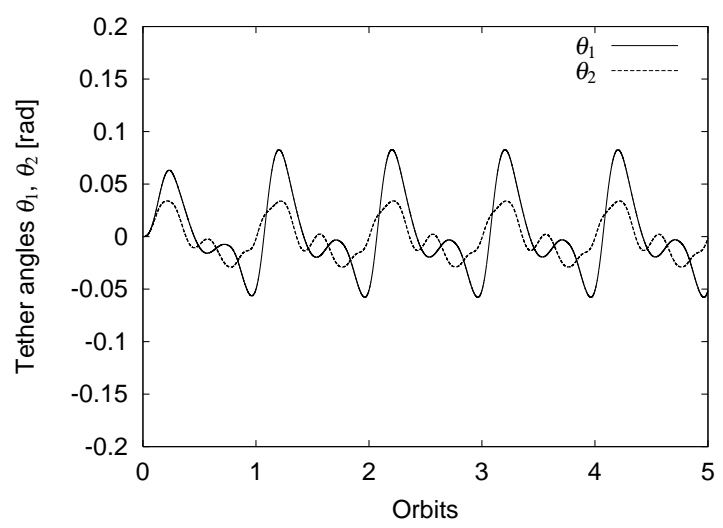

Figure 12 Time response of tether tension for PD control and $L_{1}=50-70 \mathrm{~km}$

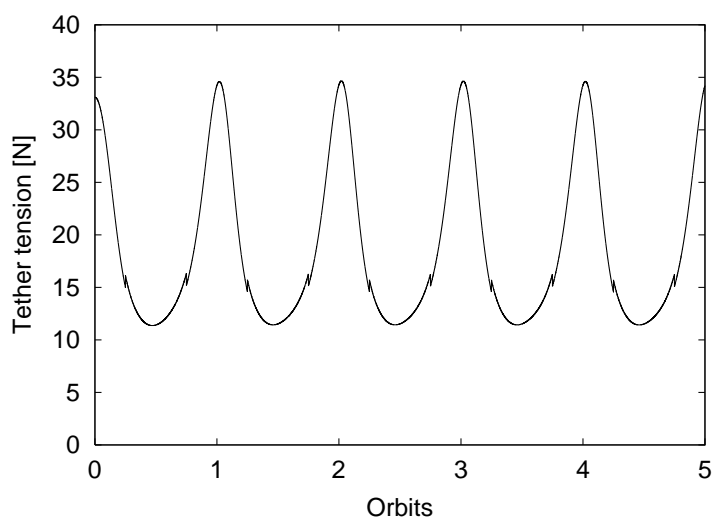

Figure 13 Time response of electric current for PD control and $L_{1}=50-70 \mathrm{~km}$

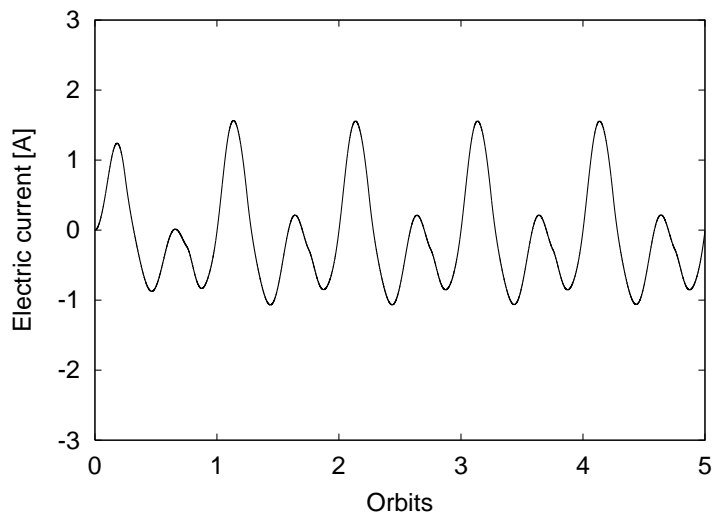


In addition, it is seen that tether 1 oscillates once per orbit for both Case-A and Case-B, whereas it oscillates twice per orbit for Case-C. This is because a tether system in an elliptic orbit is a chaotic system, and there are an infinite number of patterns of periodic motion. Furthermore, as shown in Figures 4, 8, and 12, the tether tension varies with tether length but never becomes negative, indicating the absence of slack in tether 1. For Case-B and Case-C, small rapid fluctuations of the tether tension are seen in Figures 8 and 12, respectively, whereas such changes are not observed for Case-A in Figure 4.

\section{Delayed feedback control applied to electrodynamic force}

\subsection{Delayed feedback control}

Chaotic motion is characterised by bounded variation, a sensitive dependence on the initial conditions, and indivisibility such as in regard to transitions on the phase plane. If a system is chaotic, it is generally possible for its trajectory to fall into any number of bounded regions. Ott et al. have shown that a chaotic system can be stabilised to a periodic solution with a period close to one of the bounded regions. Their method is referred to as the OGY method (Ott et al., 1990). The DF control scheme proposed by Pyragas (1992) is used to calculate the control input using both the current output and the output delayed by one period as

$$
u=K(y(t)-y(t-\tau)) .
$$

Figure 14 shows a block diagram of this method. It can be interpreted as an extension of the OGY method to feedback control for continuous systems, and has been studied as a means of stabilising the librational motion of a tethered subsatellite system to a periodic solution with the same period as that of the orbit. Very small external forces can have a large effect on the system dynamics because chaotic motion is very sensitive to the initial conditions. In other words, the magnitude of the control input for DF control becomes small when the chaotic librational motion of the system is successfully stabilised to a periodic solution. This is an advantage of DF control when applied to systems with chaotic motion. Another advantage is that the method does not require a preliminary calculation (other than the period) of the desired trajectory, making it robust with respect to parameter variations.

In this study, the following DF control is used to determine the electrodynamic force on tether 2

$$
F^{e}=K_{\mathrm{dfc}}\left(\dot{\theta}_{1}(t)-\dot{\theta}_{1}(t-\tau)\right)
$$

where $\tau$ is the orbital period. The reason why the angular velocity rather than the angle of tether 1 is used is that we care not about the librational angle but about the periodic motion, and it is known from previous studies that the tether angular rate is a suitable variable for DF control to stabilise the periodic librational motion of tether systems (Fujii et al., 2000). Because past data are required for DF control, and such data do not exist during the first orbital period in the numerical simulation, DF control is activated only after the first orbital period in the present study. 
Figure 14 Delayed feedback control method

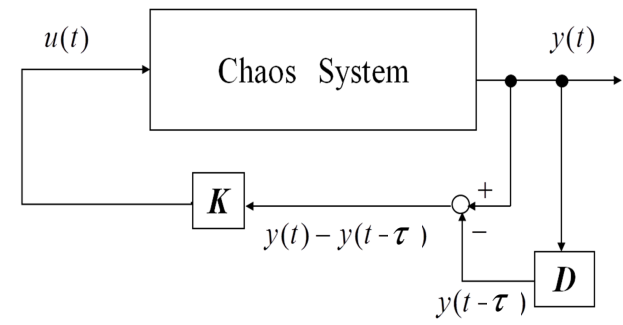

\subsection{Numerical results for DF control}

The initial states of the system were set to the same values as those for the PD control simulations, that is, $R_{0}=6,600 \mathrm{~km}, \eta=0, \theta_{1}=0 \mathrm{rad}, \theta_{2}=0 \mathrm{rad}, \dot{R}_{0}=0 \mathrm{~km} / \mathrm{s}$, $\dot{\eta}=1.2899 \times 10^{-3} \mathrm{rad} / \mathrm{s}$, and $\dot{\theta}_{1}=\dot{\theta}_{2}=0 \mathrm{rad} / \mathrm{s}$. The initial orbital period was $7457 \mathrm{~s}$, which was used as the delay time $\tau$ for DF control. The eccentricity of the centre of mass of the system was 0.2 . The DF control gain $K_{\mathrm{dfc}}$ was set to 2.0. As with the PD control simulations, three different cases were investigated with respect to $L_{1}: 50 \mathrm{~km}$ (Case-D), varying between 50 and $60 \mathrm{~km}$ (Case-E), and varying between 50 and $70 \mathrm{~km}$ (Case-F). The time responses of the tether length are omitted because they are the same as those in the previous subsection.

The time responses of the tether angles, tether tension and electric current for Case-D are shown in Figures 15 to 17, respectively. Figures 18 to 20 and 21 to 23 show the corresponding results for Case-E and Case-F, respectively. It can be seen that in all cases, the tether angles are successfully stabilised to periodic motion. For Case-D, the tether system behaves like a single pendulum because $\theta_{2}$ is almost zero, whereas nonlinear oscillations are observed in the time response of $\theta_{2}$ for Case-E and Case-F, as shown in Figures 18 and 21. In all cases, the electric current oscillates rapidly, as shown in Figures 17, 20, and 23. The magnitude of the electric current is much smaller than for the case of PD control, and decays exponentially as the tether librational motion is stabilised to a periodic motion, which is a characteristic of DF control. The decay constant is approximately 0.27 /orbit.

Figure 15 Time response of tether angles for DF control and $L_{1}=50 \mathrm{~km}$

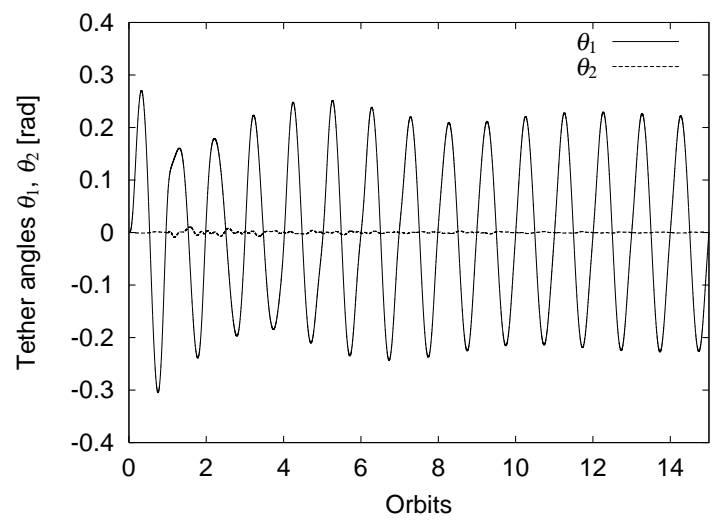


Figure 16 Time response of tether tension for DF control and $L_{1}=50 \mathrm{~km}$

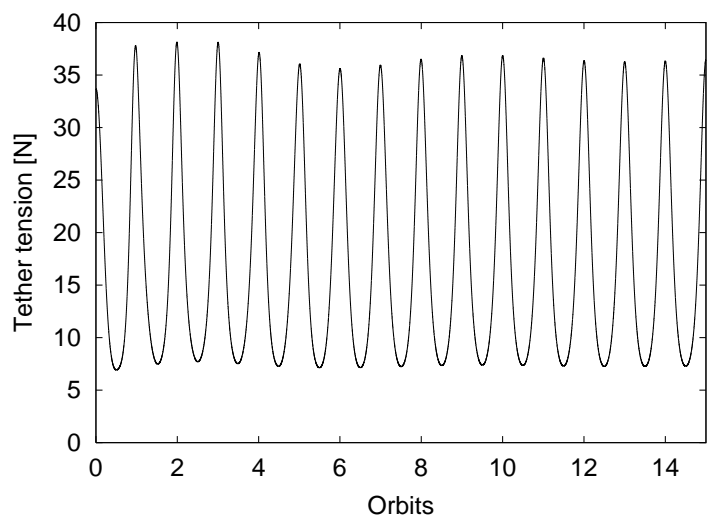

Figure 17 Time response of electric current for DF control and $L_{1}=50 \mathrm{~km}$

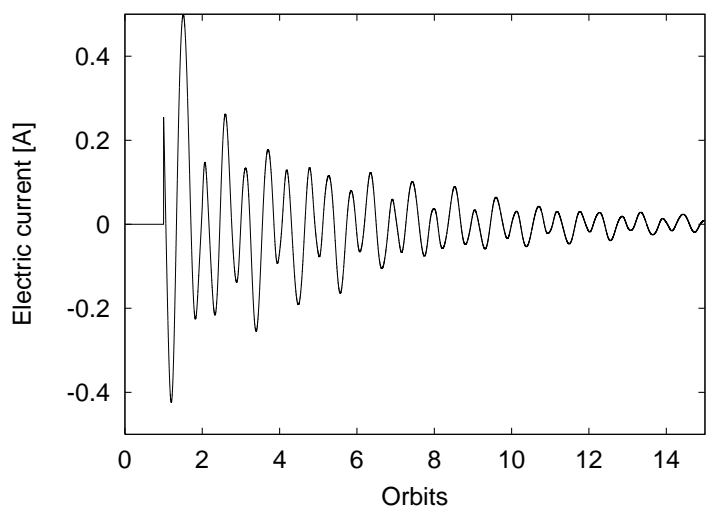

Figure 18 Time response of tether angles for DF control and $L_{1}=50-60 \mathrm{~km}$

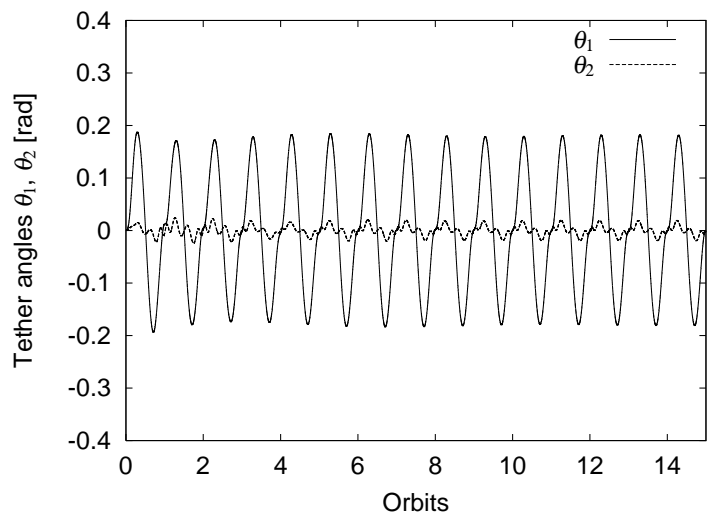


Figure 19 Time response of tether tension for DF control and $L_{1}=50-60 \mathrm{~km}$

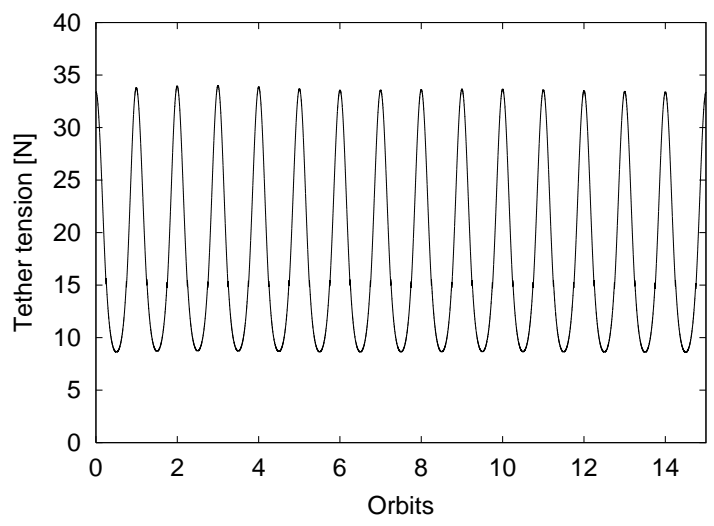

Figure 20 Time response of electric current for DF control and $L_{1}=50-60 \mathrm{~km}$

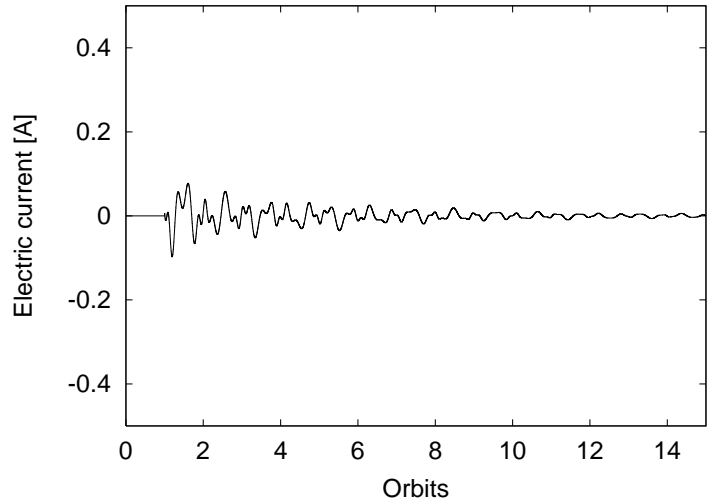

Figure 21 Time response of tether angles for DF control and $L_{1}=50-70 \mathrm{~km}$

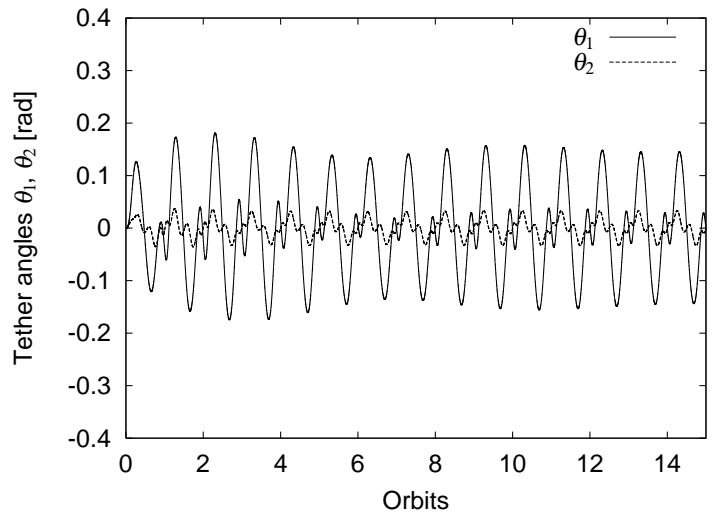


Figure 22 Time response of tether tension for DF control and $L_{1}=50-70 \mathrm{~km}$

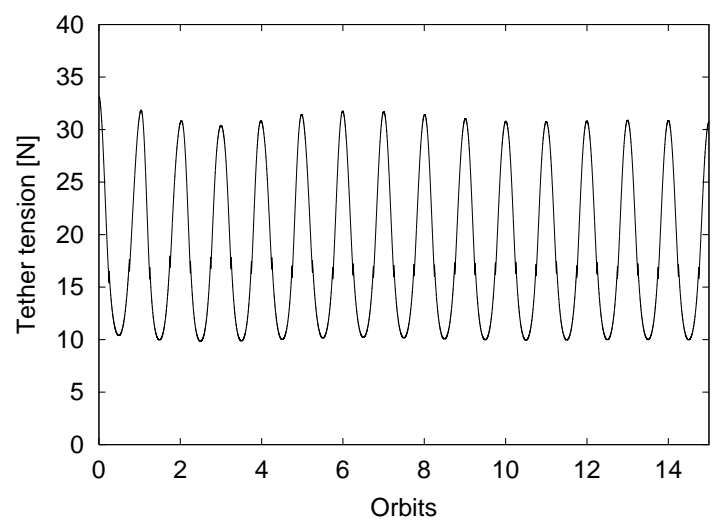

Figure 23 Time response of electric current for DF control and $L_{1}=50-70 \mathrm{~km}$

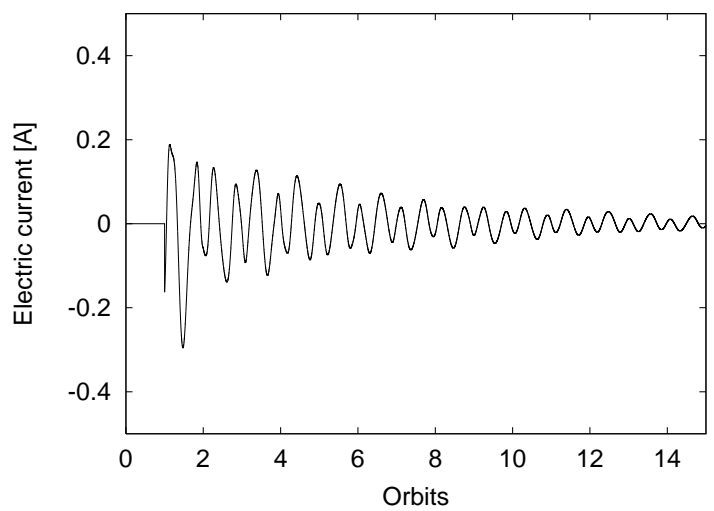

Similarly to Case-A and Case-B for PD control, the tether tension oscillates once per orbit with DF control. In addition, small rapid fluctuations in the tether tension are observed for Case-E and Case-F. As with PD control, as $L_{1}$ increases, $\theta_{1}$ decreases, the minimum tether tension increases, and the maximum tether tension decreases.

The range of oscillation of $\theta_{1}$ with DF control is greater than that with PD control. On the other hand, that of $\theta_{2}$ for Case-E and Case-F is almost the same as with PD control.

The magnitude of the electric current for Case-E is smaller than that for Case-D and Case-F. Thus, an appropriate combination of tether length and electric current control is effective in stabilising the chaotic librational in-plane motion of an EDT system in an equatorial elliptic orbit to periodic motion using a small electric current.

It should be noted that in this study only in-plane motion of the EDT system was considered, since it was assumed that the two tethers were rigid bodies without mass or inertia. However, for more realistic situations, it is necessary to take into account out-of-plane motion, tether flexibility, and inclination of the system with respect to the equator of the geomagnetic field. In order to simultaneously stabilise the in-plane and out-of-plane motion of an EDT system to periodic motion, sophisticated electric current 
control will be required, such as switching DF control (Kojima and Sugimoto, 2010). Despite this, the proposed concept based on combined electric current and tether length control is promising for stabilising librational motion of an EDT system to periodic motion with lower electric current requirements, because the geomagnetic field increases with decreasing altitude. In the near future, we will study the feasibility of this concept under more realistic EDT conditions in inclined elliptic orbits.

\section{Conclusions}

A method of controlling the libration of a new tether satellite system combining non-ETD and ETD tethers in an elliptical orbit has been proposed. The results of numerical simulations showed that the proposed scheme combining tether length and electric current control can stabilise the librational motion of the tether system to periodic motion. For the case of PD control of the ETD tether, as the range of tether length $L_{1}$ increases, the tether angle $\theta_{1}$ decreases, and the amplitude of $\theta_{2}$ increases slightly.

For the case of DF control, an appropriate combination of tether length and electric current control was found to be effective in stabilising the chaotic librational motion of a tethered satellite system in an elliptic orbit to periodic motion using a small electric current. The model used in this study, however, is very simple and does not take into account the mass or flexibility of the tether, inclination of the magnetic field, or out-of-plane motion. In future work, we plan to develop a more realistic model that considers such factors.

\section{References}

Fujii, H.A., Ichiki, W., Suda, S. and Watanabe, T.R. (2000) 'Chaos analysis on librational control of gravity-gradient satellite in elliptic orbit', Journal of Guidance, Control, and Dynamics, Vol. 23, No. 1, pp.145-146.

Hughes, P.C. (1986) Spacecraft attitude dynamics, John Wiley \& Sons, New York.

Iñarrea, M. and Peláez, J. (2010) 'Libration control of electrodynamic tethers using the extended time-delayed autosynchronization method', Journal of Guidance, Control, and Dynamics, Vol. 33, No. 3, pp.923-933.

Iñarrea, M., Lanchares, V., Pascual, A.I. and Salasa, J.P. (2014) 'Attitude stabilization of electrodynamic tethers in elliptic orbits by time-delay feedback control', Acta Astronautica, Vol. 96, No. 1, pp.280-295.

Karasopoulos, H.A. and Richardson, D.L. (1992) 'Chaos in the pitch equation of motion for the gravity-gradient satellite', AIAA Paper92-4369.

Kojima, H. and Sugimoto, T. (2009) 'Stability analysis of in-plane and out-of-plane periodic motions of electrodynamic tether system in inclined elliptic orbit', Acta Astronautica, Vol. 65, Nos. 3-4, pp.477-488.

Kojima, H. and Sugimoto, T. (2010) 'Switching delayed feedback control for an electrodynamic tether system in an inclined elliptic orbit', Acta Astronautica, Vol. 66, Nos. 7-8, pp.1072-1080.

Kojima, H., Iwasaki, M., Fujii, H.A., Blanksby, C. and Trivailo.P. (2004) 'Nonlinear control of librational motion of tethered satellites in elliptic orbits', Journal of Guidance, Control, and Dynamics, Vol. 27, No. 2, pp.229-239. 
Kojima, H., Sugimoto, Y. and Furukawa, Y. (2011a) 'Experimental study on dynamics and control of tethered satellite systems with climber', Acta Astronautica, Vol. 69, Nos. 1-2, pp.96-108.

Kojima, H., Furukawa, Y. and Trivailo, P.M. (2011b) 'Experimental verification of periodic libration of tethered satellite system in elliptic orbit', Journal of Guidance, Control, and Dynamics, Vol. 34, No. 2, pp.614-618.

Nakanishi, K., Kojima, H. and Watanabe, T. (2011) 'Trajectories of in-plane periodic solutions of tethered satellite system projected on van der Pol planes', Acta Astronautica, Vol. 68, Nos. 7-8, pp.1024-1030.

Ott, E., Grebogi, C. and Yorke, J.A. (1990) 'Controlling chaos', Physical Review Letters, Vol. 64, No. 11, pp.1196-1199.

Peláez, J. and Andres, Y.N. (2005) 'Dynamics stability of electrodynamic tethers in inclined elliptical orbits', Journal of Guidance, Control, and Dynamics, Vol. 28, No. 4, pp.611-622.

Peláez, J. and Lara, M. (2003) 'Periodic solutions in rigid electrodynamic tethers on inclined orbits', Journal of Guidance, Control, and Dynamics, Vol. 26, No. 3, pp.395-406.

Peláez, J. and Lorenzini, E.C. (2005) 'Libration control of electrodynamic tethers in inclined orbits', Journal of Guidance, Control, and Dynamics, Vol. 28, No. 2, pp.269-279.

Pyragas, K. (1992) 'Continuous control of chaos by self-controlling feedback', Physics Letters A, Vol. 170 , No. 6, pp.421-428.

Williams, P. (2006) 'Energy rate feedback for libration control of electrodynamic tethers', Journal of Guidance, Control, and Dynamics, Vol. 29, No. 1, pp.221-223.

\section{Appendix A}

Mathematica code for deriving equations of motion

The Mathematica code used to derive the equations of motion [equation (12)] is presented below.

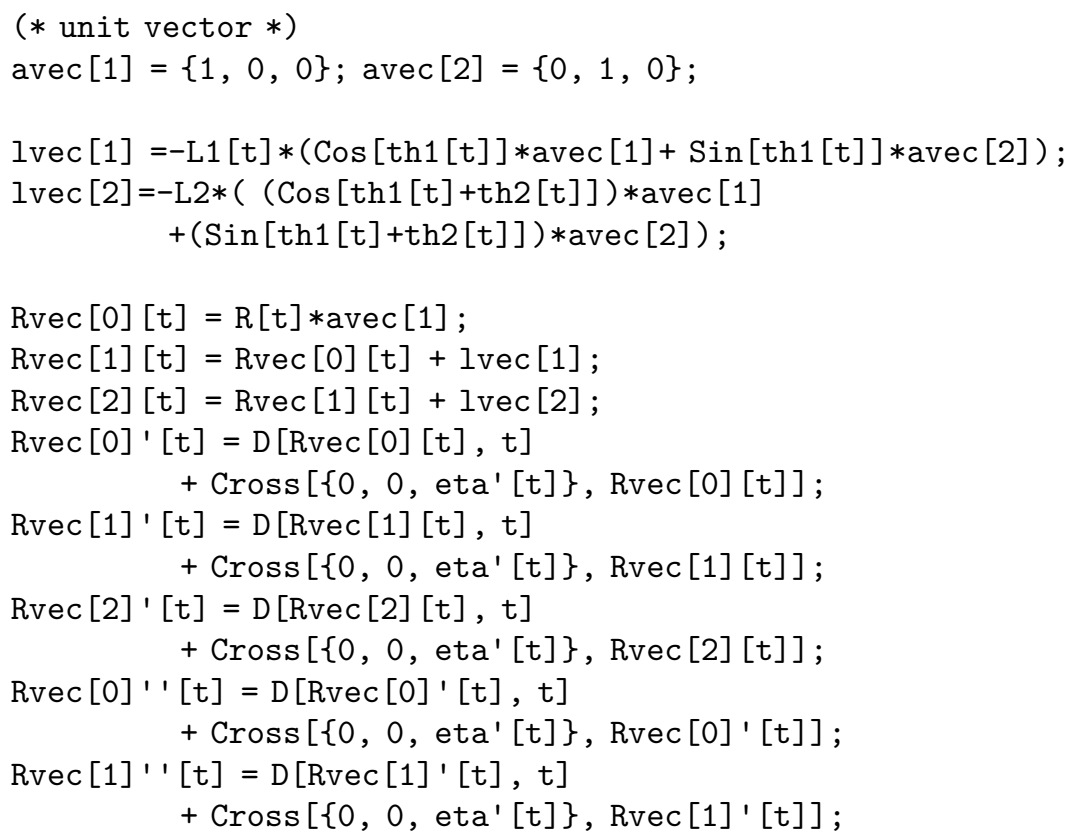




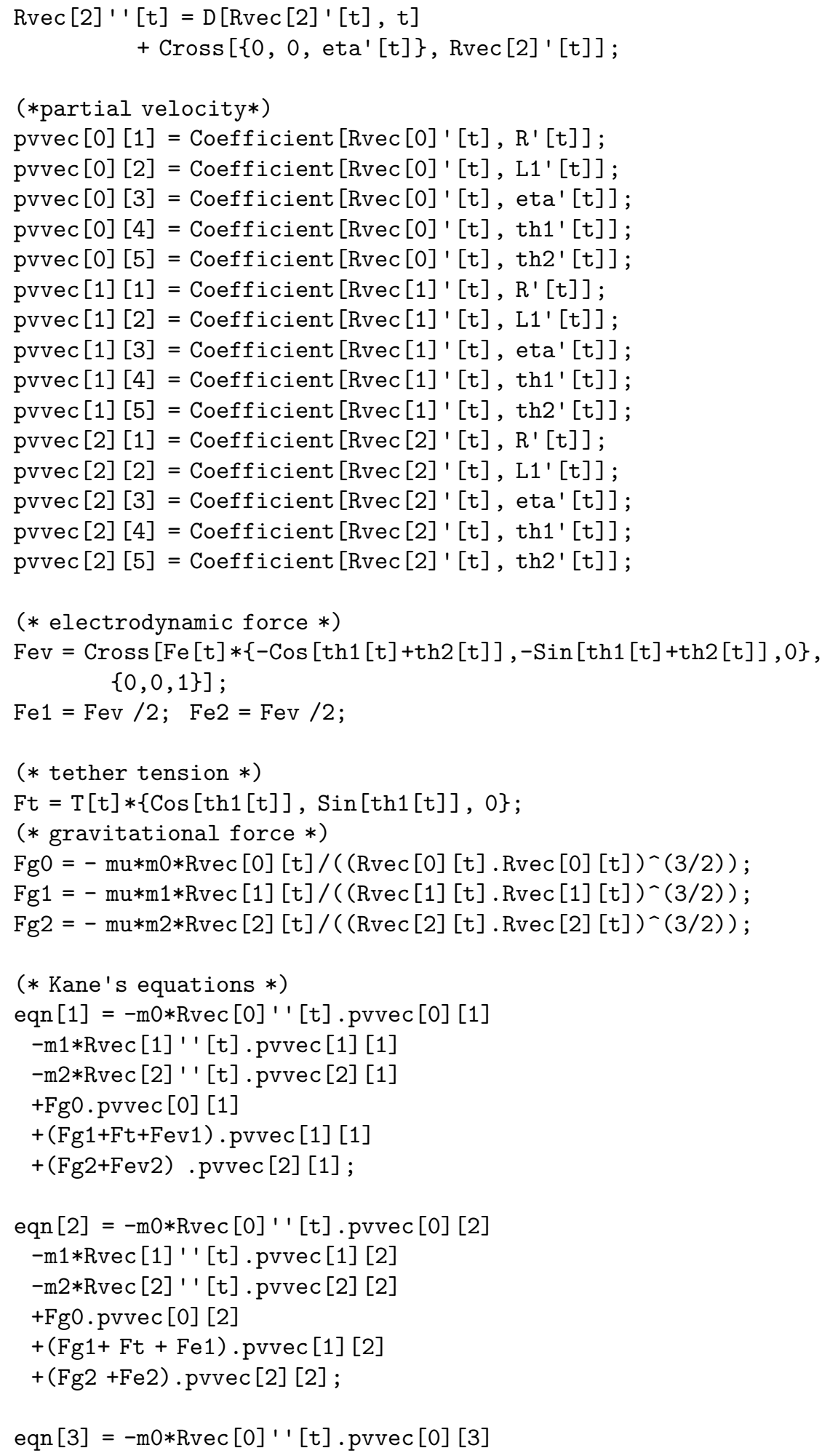




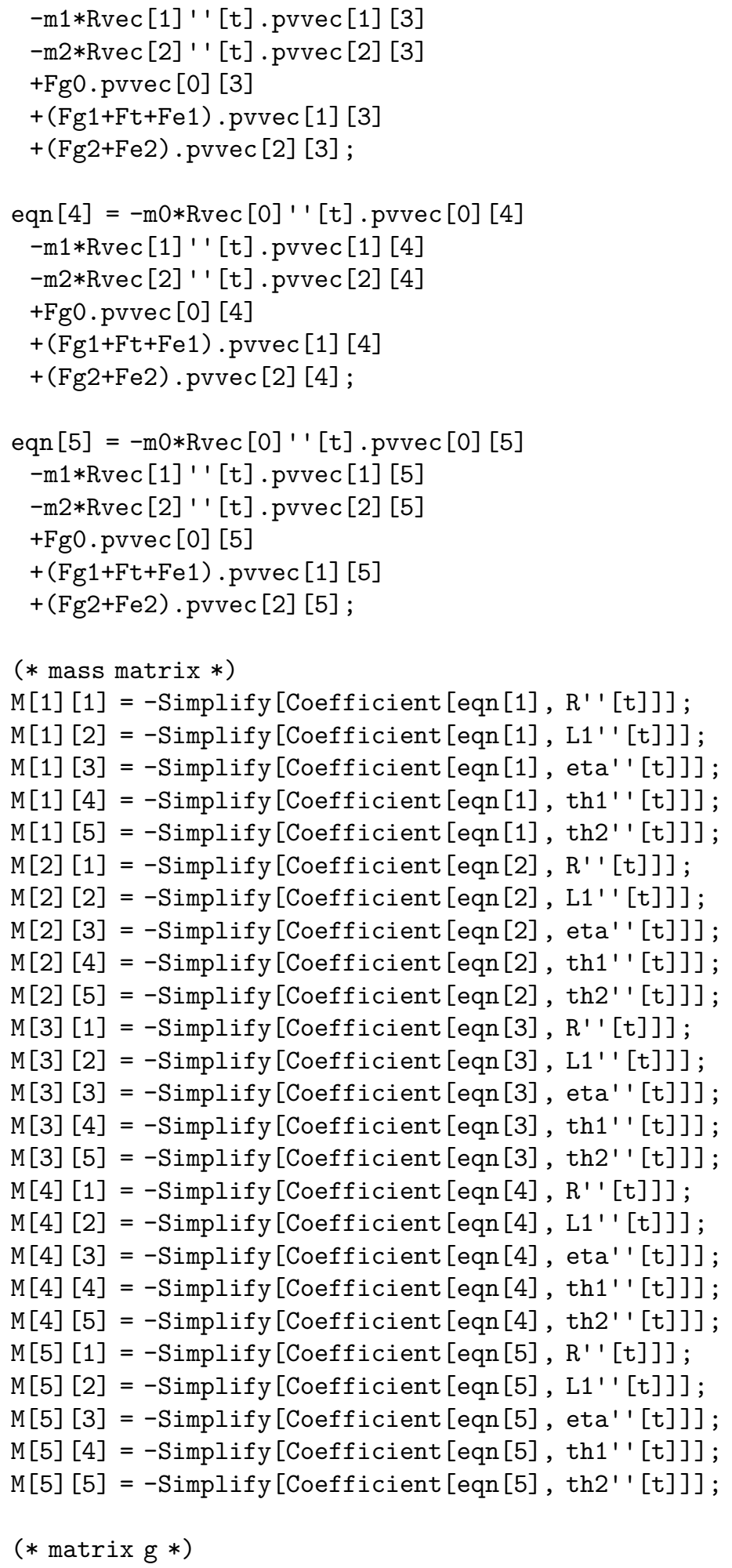




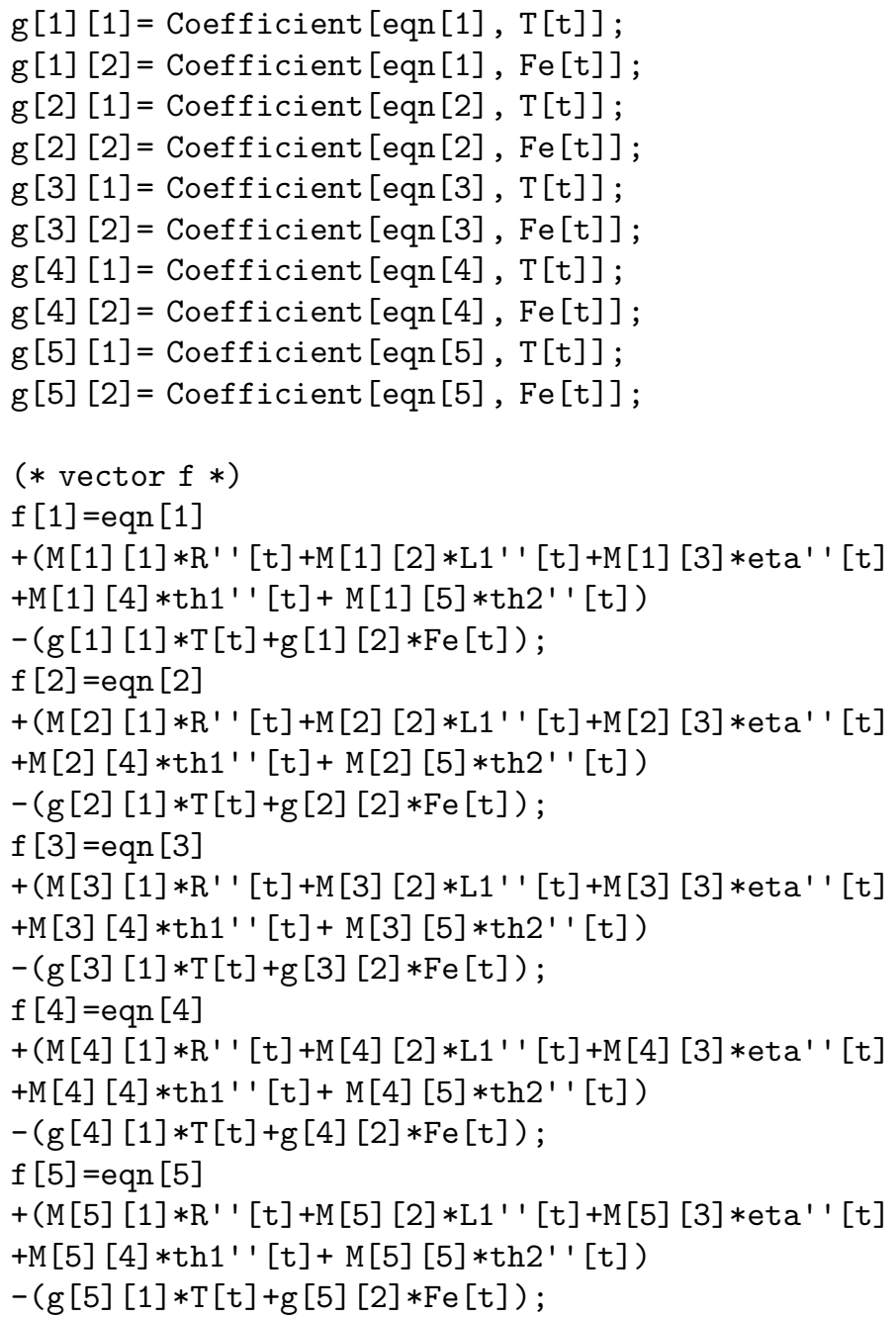

As an example, using this code, the first row elements of the mass matrix are obtained as:

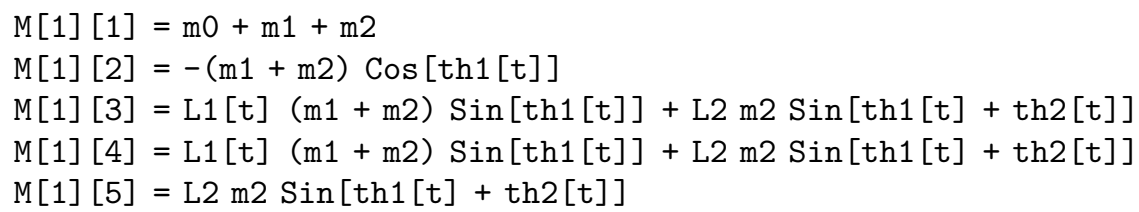

\section{Appendix B}

Elements of mass matrix and nonlinear terms for equation of motion

Using the Mathematica code in Appendix A, the elements of the mass matrix $\boldsymbol{M}=M_{i j}\left(=M_{j i}\right),(i, j=1, \cdots, 5), \boldsymbol{f}=\left[f_{1}, f_{2}, f_{3}, f_{4}, f_{5}\right]^{T}$, and $\boldsymbol{g}_{j},(j=1,2)$ are 
obtained as follows:

$$
\begin{aligned}
& M_{11}=m_{0}+m_{1}+m_{2} \\
& M_{12}=-\left(m_{1}+m_{2}\right) \cos \theta_{1} \\
& M_{13}=\left(m_{1}+m_{2}\right) L_{1} \sin \theta_{1}+m_{2} L_{2} \sin \left(\theta_{1}+\theta_{2}\right) \\
& M_{14}=\left(m_{1}+m_{2}\right) L_{1} \sin \theta_{1}+m_{2} L_{2} \sin \left(\theta_{1}+\theta_{2}\right) \\
& M_{15}=m_{2} L_{2} \sin \left(\theta_{1}+\theta_{2}\right) \\
& M_{22}=m_{1}+m_{2} \\
& M_{23}=-\left(m_{1}+m_{2}\right) R \sin \theta_{1}-m_{2} L_{2} \sin \theta_{2} \\
& M_{24}=-m_{2} L_{2} \sin \theta_{2} \\
& M_{25}=-m_{2} L_{2} \sin \theta_{2} \\
& M_{33}=\left(m_{0}+m_{1}+m_{2}\right) R^{2}+\left(m_{1}+m_{2}\right) L_{1}^{2}+m_{2} L_{2}^{2}-2\left(m_{1}+m_{2}\right) L_{1} R \cos \theta_{1} \\
& +2 m_{2} L_{1} L_{2} \cos \theta_{2}-2 m_{2} L_{2} R \cos \left(\theta_{1}+\theta_{2}\right) \\
& M_{34}=\left(m_{1}+m_{2}\right) L_{1}^{2}-\left(m_{1}+m_{2}\right) L_{1} R \cos \theta_{1}+2 m_{2} L_{1} L_{2} \cos \theta_{2} \\
& -m_{2} L_{2}\left(-L_{2}+R \cos \left(\theta_{1}+\theta_{2}\right)\right) \\
& M_{35}=m_{2} L_{2}\left(L_{2}+L_{1} \cos \theta_{2}-R \cos \left(\theta_{1}+\theta_{2}\right)\right) \\
& M_{44}=\left(m_{1}+m_{2}\right) L_{1}^{2}+m_{2} L_{2}^{2}+2 m_{2} L_{1} L_{2} \cos \theta_{2} \\
& M_{45}=m_{2} L_{2}\left(L_{1} \cos \theta_{2}+L_{2}\right) \\
& M_{55}=m_{2} L_{2}^{2} \\
& \boldsymbol{g}_{1}=\left[\begin{array}{c}
\cos \theta_{1} \\
-1 \\
R \sin \theta_{1} \\
0 \\
0
\end{array}\right] \\
& \boldsymbol{g}_{2}=\left[\begin{array}{c}
-\sin \left(\theta_{1}+\theta_{2}\right) \\
\sin \theta_{2} \\
-\left(L_{2} / 2\right)-L_{1} \cos \theta_{2}+R \cos \left(\theta_{1}+\theta_{2}\right) \\
-\left(L_{2} / 2\right)-L_{1} \cos \theta_{2} \\
-L_{2} / 2
\end{array}\right] \\
& f_{1}=\left(m_{0}+m_{1}+m_{2}\right) R \dot{\eta}^{2}-\left(m_{1}+m_{2}\right) L_{1}\left(\dot{\eta}+\dot{\theta}_{1}\right)^{2} \cos \theta_{1} \\
& -m_{2} L_{2}\left(\dot{\eta}+\dot{\theta}_{1}+\dot{\theta}_{2}\right)^{2} \cos \left(\theta_{1}+\theta_{2}\right)-2\left(m_{1}+m_{2}\right) \dot{L}_{1}\left(\dot{\eta}+\dot{\theta}_{1}\right) \sin \theta_{1} \\
& -\frac{\mu m_{0}}{R^{2}}-\frac{\mu m_{1}\left(R-L_{1} \cos \theta_{1}\right)}{\left(R^{2}+L_{1}^{2}-2 L_{1} R \cos \theta_{1}\right)^{(3 / 2)}} \\
& -\frac{\mu m_{2}\left\{R-L_{1} \cos \theta_{1}-L_{2} \cos \left(\theta_{1}+\theta_{2}\right)\right\}}{\left\{R^{2}+L_{1}^{2}+L_{2}^{2}-2 L_{1} R \cos \theta_{1}+2 L_{1} L_{2} \cos \theta_{2}\right.} \\
& \left.-2 L_{2} R \cos \left(\theta_{1}+\theta_{2}\right)\right\}^{(3 / 2)} \\
& f_{2}=-\left(m_{1}+m_{2}\right) R \dot{\eta}^{2} \cos \theta_{1}+\left(m_{1}+m_{2}\right) L_{1}\left(\dot{\eta}+\dot{\theta}_{1}\right)^{2}
\end{aligned}
$$




$$
\begin{aligned}
& +{ }^{`} m_{2} L_{2}\left(\dot{\eta}+\dot{\theta}_{1}+\dot{\theta}_{2}\right)^{2} \cos \theta_{2}+2\left(m_{1}+m_{2}\right) \dot{R} \dot{\eta} \sin \theta_{1} \\
& +\frac{\mu m_{1}\left(R \cos \theta_{1}-L_{1}\right)}{\left(R^{2}+L_{1}^{2}-2 L_{1} R \cos \theta_{1}\right)^{(3 / 2)}} \\
& +\frac{\mu m_{2}\left(R \cos \theta_{1}-L_{1}-L_{2} \cos \theta_{2}\right)}{\left\{R^{2}+L_{1}^{2}+L_{2}^{2}-2 L_{1} R \cos \theta_{1}+2 L_{1} L_{2} \cos \theta_{2}\right.} \\
& \left.-2 L_{2} R \cos \left(\theta_{1}+\theta_{2}\right)\right\}^{(3 / 2)} \\
& f_{3}=-\left(m_{1}+m_{2}\right) R L_{1} \dot{\theta}_{1}^{2} \sin \theta_{1}-m_{2} R L_{2}\left(\dot{\theta}_{1}+\dot{\theta}_{2}\right)^{2} \sin \left(\theta_{1}+\theta_{2}\right) \\
& +m_{2} L_{1} L_{2} \dot{\theta}_{2}^{2} \sin \theta_{2}-2\left(m_{0}+m_{1}+m_{2}\right) R \dot{R} \dot{\eta} \\
& +2\left(m_{1}+m_{2}\right) \dot{R} L_{1} \dot{\eta} \cos \theta_{1}-2\left(m_{1}+m_{2}\right) R L_{1} \dot{\eta} \dot{\theta}_{1} \sin \theta_{1} \\
& +2\left(m_{1}+m_{2}\right)\left(R \cos \theta_{1}-L_{1}\right) \dot{L}_{1}\left(\dot{\eta}+\dot{\theta}_{1}\right) \\
& -2 m_{2} \dot{L}_{1} L_{2}\left(\dot{\eta}+\dot{\theta}_{1}\right) \cos \theta_{2}+2 m_{2} L_{1} L_{2}\left(\dot{\eta}+\dot{\theta}_{1}\right) \dot{\theta}_{2} \sin \theta_{2} \\
& +2 m_{2} \dot{R} L_{2} \dot{\eta} \cos \left(\theta_{1}+\theta_{2}\right)-2 m_{2} R L_{2} \dot{\eta}\left(\dot{\theta}_{1}+\dot{\theta}_{2}\right) \sin \left(\theta_{1}+\theta_{2}\right) \\
& f_{4}=\left(m_{1}+m_{2}\right) R L_{1} \dot{\eta}^{2} \sin \theta_{1}+m_{2} L_{1} L_{2} \dot{\theta}_{2}^{2} \sin \theta_{2}+m_{2} R L_{2} \dot{\eta}^{2} \sin \left(\theta_{1}+\theta_{2}\right) \\
& -2\left(m_{1}+m_{2}\right) L_{1} \dot{L}_{1}\left(\dot{\eta}+\dot{\theta}_{1}\right)+2\left(m_{1}+m_{2}\right) \dot{R} L_{1} \dot{\eta} \cos \theta_{1} \\
& +2 m_{2} L_{1} L_{2}\left(\dot{\eta}+\dot{\theta}_{1}\right) \dot{\theta}_{2} \sin \theta_{2}-2 m_{2} \dot{L}_{1} L_{2}\left(\dot{\eta}+\dot{\theta}_{1}\right) \cos \theta_{2} \\
& +2 m_{2} \dot{R} L_{2} \dot{\eta} \cos \left(\theta_{1}+\theta_{2}\right)-\frac{\mu m_{1} R L_{1} \sin \theta_{1}}{\left(R^{2}+L_{1}^{2}-2 L_{1} R \cos \theta_{1}\right)^{(3 / 2)}} \\
& -\frac{\mu m_{2} R\left\{L_{1} \sin \theta_{1}+L_{2} \sin \left(\theta_{1}+\theta_{2}\right)\right\}}{\left\{R^{2}+L_{1}^{2}+L_{2}^{2}-2 L_{1} R \cos \theta_{1}+2 L_{1} L_{2} \cos \theta_{2}\right.} \\
& \left.-2 L_{2} R \cos \left(\theta_{1}+\theta_{2}\right)\right\}^{(3 / 2)} \\
& f_{5}=-m_{2} L_{1} L_{2}\left(\dot{\eta}+\dot{\theta}_{1}\right)^{2} \sin \theta_{2}+m_{2} R L_{2} \dot{\eta}^{2} \sin \left(\theta_{1}+\theta_{2}\right) \\
& \text { - } 2 m_{2} \dot{L}_{1} L_{2}\left(\dot{\eta}+\dot{\theta}_{1}\right) \cos \theta_{2}+2 m_{2} \dot{R} L_{2} \dot{\eta} \cos \left(\theta_{1}+\theta_{2}\right) \\
& -\frac{\mu m_{2} L_{2}\left\{R \sin \left(\theta_{1}+\theta_{2}\right)-L_{1} \sin \theta_{2}\right\}}{\left\{R^{2}+L_{1}^{2}+L_{2}^{2}-2 L_{1} R \cos \theta_{1}+2 L_{1} L_{2} \cos \theta_{2}\right.} \\
& \left.-2 L_{2} R \cos \left(\theta_{1}+\theta_{2}\right)\right\}^{(3 / 2)}
\end{aligned}
$$

\begin{tabular}{|c|c|}
\hline $\boldsymbol{F}^{e}$ & electrodynamic force vector \\
\hline $\boldsymbol{F}_{i}^{g}$ & gravitational force affecting the $i^{\text {th }}$ satellite \\
\hline & electric current in tether $2, \mathrm{~A}$ \\
\hline$L_{1}, L_{2}$ & lengths of tethers 1 and 2 , respectively ( $L_{1}=$ varing, $\left.L_{2}=50 \mathrm{~km}\right)$ \\
\hline$m_{0}, m_{1}, m_{2}$ & $\begin{array}{l}\text { masses of mother satellite and subsatellites } 1 \text { and } 2 \text {, respectively } \\
\left(m_{0}=10,000 \mathrm{~kg}, m_{1}=m_{2}=50 \mathrm{~kg}\right)\end{array}$ \\
\hline $\boldsymbol{R}_{i}$ & position of the $i^{\text {th }}$ satellite in the orbital reference frame \\
\hline & $j^{\text {th }}$ partial velocity of the $i^{\text {th }}$ satellite \\
\hline & state variables $\left(=\left[R_{0}, L_{1}, \eta, \theta_{1}, \theta_{2}, \dot{R}_{0}, \dot{L}_{1}, \dot{\eta}, \dot{\theta}_{1}, \dot{\theta}_{2}\right]^{T}\right)$ \\
\hline
\end{tabular}

\section{Nomenclature}




$\begin{array}{ll}K_{\mathrm{dfc}} & \text { DF control gain } \\ K_{P}, K_{D} & \text { PD control gains } \\ \eta & \text { true anomaly for the mother satellite, rad } \\ \theta_{1}, \theta_{2} & \text { in-plane angles of tethers 1 and 2, respectively, rad } \\ \psi_{m}, \theta_{m} & \text { latitude and longitude, respectively, in the geocentric } \\ & \text { inertial reference frame, rad } \\ \omega & \text { angular velocity of the orbital reference frame, rad } / \mathrm{s} \\ \mu_{m} & \text { magnetic moment of Earth's dipole }=8.1 \times 10^{15} \mathrm{~Wb} \cdot \mathrm{m} \\ \mu_{e} & \text { gravitational constant of Earth }=3.98613 \times 10^{5} \mathrm{~km} / \mathrm{s}^{2} \\ \tau & \text { delay time for DF control. }\end{array}$

